\title{
Analysis of OCT Images to Optimize Glaucoma Diagnosis
}

\author{
Nahida Akter ${ }^{1}$, Jack Phu ${ }^{1,2}$, Stuart Perry ${ }^{3}$, John Fletcher ${ }^{4}$, Michael Kalloniatis ${ }^{1,2}$, Maitreyee Roy ${ }^{1}$ \\ ${ }^{1}$ School of Optometry and Vision Science, UNSW Sydney, NSW 2052, Australia \\ ${ }^{2}$ Center for Eye Health, UNSW Sydney, NSW 2052, Australia \\ ${ }^{3}$ School of Electrical and Data Engineering, University of Technology Sydney, NSW 2007, Australia \\ ${ }^{4}$ School of Electrical Engineering and Telecommunications, UNSW Sydney, NSW 2052, Australia \\ Author e-mail address: Maitreyee.roy@unsw.edu.au
}

\begin{abstract}
In this paper, data from OCT images are extracted, statistically analyzed and further an image processing task has been performed on optic nerve head images to optimize features used in the diagnosis of glaucoma.

OCIS codes: (170.0170) Medical optics and biotechnology; (170.0110) Imaging systems;

(170.4500) Optical coherence tomography
\end{abstract}

\section{Introduction}

Glaucoma is an eye disease where retinal ganglion cell (RGC) death occurs, manifesting clinically as observable anatomical changes at the optic nerve head $(\mathrm{ONH})$ [1]. There is a need to optimize the parameters driving glaucoma diagnosis. At both detection and progression stages the diagnosis process does not depend only on one or two variables, but rather a Bayesian approach combining several parameters [2]. The current clinical standard method of diagnosing glaucoma is a complete eye exam, with a slew of data which can be clinically challenging to interpret. Furthermore, given the significant overlap in ocular measurements between normal subjects and patients with very early glaucoma, there is interest in developing complementary techniques to assist in distinguishing true pathology from normal ageing changes, such as artificial intelligence systems [3].

Imaging technologies such as optical coherence tomography (OCT) play a significant role in glaucoma diagnosis, progression monitoring and quantification of structural damage [4]. Commercially available spectral domain (SD) OCT instruments have potential in assessing glaucoma due to their non-invasive, non-contact imaging, high axial resolution and faster scanning process. Though characteristic observable changes occurring in glaucoma such as enlarged cup-disc ratio have been well-accepted, a question remains regarding the integration of data from extra dimensions obtainable using SD-OCT, and whether extra-dimensional data can assist in separating disease from normal ageing.

In this study, we aimed to optimize glaucoma diagnosis parameters extracted from OCT images of real patient data. Our initial work is to identify the significant features to detect glaucomatous changes and then we introduced a new region of interest (ROI) extraction and measurements from B-scan of $\mathrm{ONH}$ images to improve the diagnosis of glaucoma.

\section{Data and Methodology}

Clinical data from two subject groups of patients seen at the Centre for Eye Health were analysed: $n=100$ consisting of 50 normal subjects and 50 glaucoma patients. The data were extracted from the Cirrus HD-OCT (Carl Zeiss Meditec) and 11 features (age, gender, average retinal nerve fibre layer (RNFL) thickness, cup to disc ratio (CDR), corneal thickness, intraocular pressure (IOP), mean deviation (MD), pattern standard deviation (PSD), spherical equivalent refractive error (SE), visual field index (VFI) and family history were extracted and transferred into Microsoft Excel. We performed independent t-test and ROC curve analysis to find the possible parameters and examined the separability of their distributions using sensitivity index d-prime (d'). Secondly, based on knowledge regarding known anatomical changes occurring at the $\mathrm{ONH}$ in glaucoma, we performed a pilot study to extract a new ROI from 40 ONH B-scan images retina (Spectralis OCT, Heidelberg Engineering) for both normal and glaucoma patients. The ROI was extracted from MATLAB R2018a and the area, mean area, standard deviation was calculated using the Image J software.

\section{Results and Discussion}

Fig. 1 reveals that among 11 parameters of glaucoma detection only age, RNFL, CDR, MD, PSD and VFI are found statistically significant $(\mathrm{p}<0.0001)$ in glaucoma patients when compared with normal. Furthermore, the area under ROC curve was $>0.7$ for all of these significant parameters (Table 1), however only age and RNFL had a high separability of d' $=1.38$ and 1.44 respectively. This result encouraged us to develop new parameters from the structural anatomy of the ONH. Previous studies have calculated the cup and disk area with some limitations [5], but here we 
calculated the total mean area of ONH surface, and our initial point of ROI selection was measured from disk to cup with minimum rim width demonstrated in Fig. 2(a) and all final images in Fig. 2(b).

Table 1. Area under the ROC curve for all parameters

\begin{tabular}{ccc}
\hline Parameters & Area & Std. Error \\
\hline Age & 0.944 & 0.024 \\
CDR & 0.714 & 0.051 \\
Corneal_thickness & 0.446 & 0.058 \\
IOP & 0.506 & 0.058 \\
PSD & 0.856 & 0.038 \\
SE & 0.493 & 0.059 \\
Family_history & 0.500 & 0.058 \\
RNFL_thickness & 0.939 & 0.022 \\
VFI & 0.853 & 0.038 \\
MD & 0.758 & 0.050 \\
Gender & 0.590 & 0.057 \\
\hline & & \\
\hline
\end{tabular}

(a)

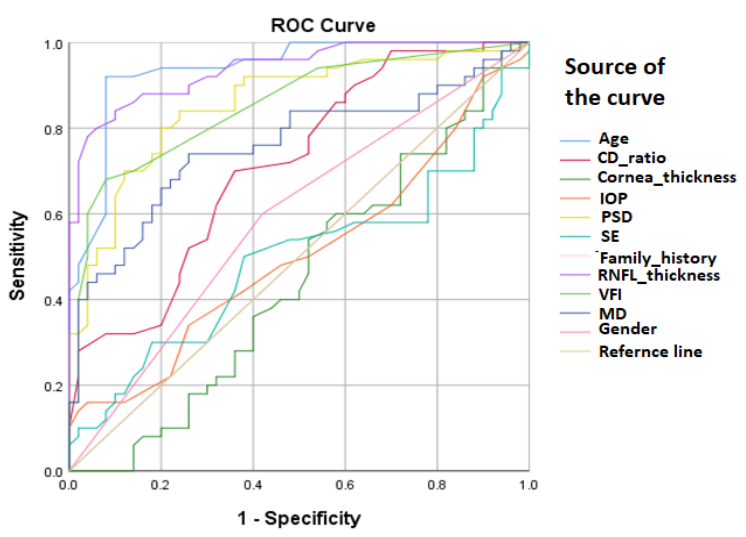

Fig. 1. ROC curve analysis for all parameters

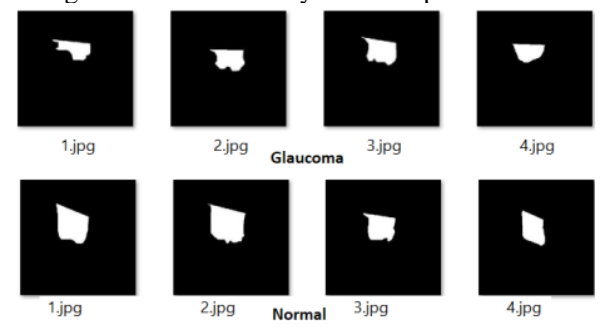

(b)

Fig. 2. (a) ROI selection and extraction from B-scan (b) Final ROI extraction images for glaucoma and normal

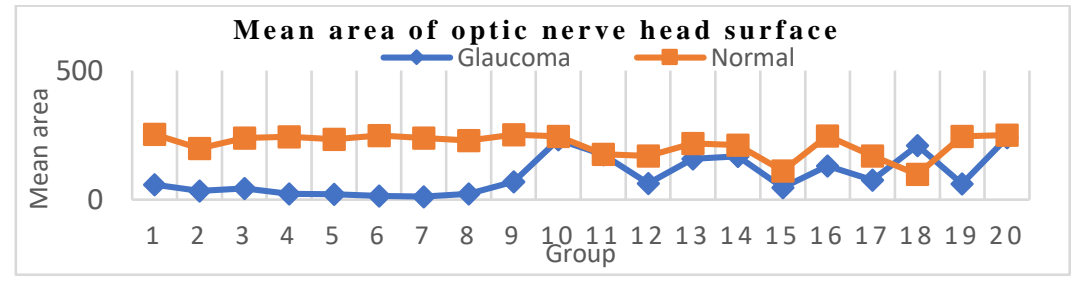

Fig. 3. The mean area of $\mathrm{ONH}$ surface for normal and glaucoma patient

Our study showed the mean area of ONH surface area to be lower for glaucoma than normal (Fig.3) while the deformation is higher in glaucoma patient in comparison with normal.

\section{Conclusion}

Age and RNFL thickness appear to distinguish between glaucoma and normal subjects in comparison to CDR, MD, PSD and VFI. Furthermore, the pilot study showed that high values of ONH surface deformation and low values of mean area can also be useful for glaucoma diagnosis, which suggests that cup and disk parameters such as CDR may benefit from further detailed analysis to improve separability.

\section{References}

[1] Davis, B.M., Crawley, L., Pahlitzsch, M., Javaid, F., and Cordeiro, M.F., "Glaucoma: the retina and beyond,” Acta Neuropathol 132(6), 807826 (2016).

[2] Al-Aswad, L.A., “Artificial Intelligence, Big Data, and Medical Analytics,” Glaucoma Today,” Bryn Mawr Communications, 45-48 (2017). [3] Liu, S., FRANZCO, S. L. G., Schulz, A., Kalloniatis, M., Zangerl, B., Cai, W., Gao, Y., FRANZCO, B. C., FRANZCO, H. A., Grigg, J., FRANZCO, Chu, D., Klistorner, A., "A deep learning-based algorithm identifies glaucomatous discs using monoscopic fundus photographs," Ophthalmology Glaucoma 1(1), 15-22 (2018).

[4] Greenfield, D.S., and Weinreb, R.N., "Role of optic nerve imaging in glaucoma clinical practice and clinical trials," Am J Ophthalmol.145(4), 598-603 (2008).

[5] Takada, N., Omodaka, K., Kikawa, T., Takagi, A., Matsumoto, A., Yokoyama, Y., Shiga, Y., Maruyama, K., Takahashi, H., Akiba, M., and Nakazawa, T., "OCT-based quantification and classification of optic disc structure in glaucoma patients," PloS one 11(8), 1-16 (2016). 